\title{
Contextual Control of Trigeminal Sensorimotor Function
}

\author{
Kent C. Berridge and John C. Fentress \\ Department of Psychology, Dalhousie University, Halifax, Nova Scotia B3H 4J1, Canada
}

\begin{abstract}
Simple actions, such as rhythmic tongue protrusions, forelimb facial strokes, and forelimb flails, are emitted by rats both during taste-elicited ingestion/aversion and during postprandial grooming. This study combined peripheral trigeminal deafferentation with a computer-assisted video analysis of action form to examine the use of cutaneous feedback from the face in action production. Changes in action form after deafferentation were found to be context-dependent: Deformations characterized rhythmic tongue protrusions when emitted in ingestive but not in grooming contexts. The opposite was true for alterations in forelimb action. Further, postprandial grooming as a whole was found to comprise distinct sequentially defined phases. Actions occurring in one highly stereotyped sequence phase were protected from deafferentation effects, although the same actions occurring outside of this phase were not. The results suggest that behavioral context (e.g., gronming versus ingestive set, sequence phase) can shift the integration of sensory guided and endogenous mechanisms that pattern simple actions.
\end{abstract}

The study of simple action patterns in invertebrate and vertebrate species has contributed greatly to our understanding of the neural mechanisms of behavioral control. A major theme in the study of simple action has been the degree to which the pattern of action is controlled by the pattern of the afferent signal (reflex action) versus the degree to which the behavioral output contains elements of form not present in the input (endogenous pattern generation).

Sherrington's (1906) definition of the reflex as the unit reaction in nervous integration provided a powerful conceptual model of simple behavior. From the gill withdrawal of the invertebrate Aplysia (Pinsker et al., 1970) to the muscle stretch (Gardner, 1968), eyeblink (Kugelberg, 1952), and pinna (Sherrington, 1906) reflexes of many vertebrates, simple action in response to a specific signal may constitute a fundamental unit of behavior (Gallistel, 1980). Conversely, many clear demonstrations exist of action in which the behavioral pattern cannot be traced to patterns in the afferent signal, but instead must arise from endogenous oscillators and cell networks. The oscillations of locust flight (Wilson, 1961), leech swimming (Stent et al., 1978), the form of command cell-triggered startle responses of many invertebrates and even of teleost fish (Kimmel and Eaton, 1976), and the pattern of mammalian swallowing (Doty, 1976), are all examples of action whose pattern arises, in varying degrees, from central specifications. Of course, examples of pure reflexes or endogenously generated patterns are exceedingly rare; far more

Received Apr. 11, 1985; revised June 10, 1985; accepted July 3, 1985.

This work was supported by postdoctoral fellowships from NATO and the Killam Foundation to K.C.B., and by grants from the Canadian MRC and NSERC to J.C.F. We thank W. G. Danilchuk and H. Parr for assistance with the analysis and programming, and M. F. Jacquin for demonstrating the deafferentation procedure.

Correspondence should be addressed to Dr. Berridge at his present address: Department of Psychology, Neuroscience Laboratory Bldg., The University of Michigan, Ann Arbor, MI 48109.

Copyright (C) 1986 Society for Neuroscience $0270-6474 / 86 / 020325-06 \$ 02.00 / 0$ common are intermediate instances (including many of the examples above), in which afferent signals trigger an action but central generators dictate the pattern, or in which reflex mechanisms and pattern generators converge to create a complex action. In addition, a variety of conceptual systems by which incoming stimuli could interact in a graded fashion with endogenous "motor tapes" or "sensory tapes" have been suggested (cf. Ewert, 1985; Hoyle, 1964).

To assign any action solely to a reflex, an endogenously generated, or an intermediate category presupposes that actions are essentially static entities, that once a movement pattern has been analyzed under a given set of circumstances it can be counted on to continue to operate by the same principles whenever it is emitted. A variety of evidence exists, however, to suggest that the rules of neural stimulus processing and action production are not static, but rather vary systematically under specifiable conditions. Chapin and Woodward (1981), for example, found the response of cortical somatosensory units (S1) to cutaneous stimuli to be suppressed during the performance of rapid actions. Similar phenomena have been reported in a variety of vertebrate and invertebrate species (see Evarts et al., 1984; Fentress, 1984, for reviews). In a slightly different vein, sensory processes involved in behavior may be influenced by contextual factors: For example, Manetto and Lidsky (1984) have recently reported that the sensitivity to perioral tactile stimuli of neostriatal single units is enhanced in cats that are visually tracking food.

Changes in the neural processing of sensory signals during different phases of action, or under different contextual conditions, raise the possibility that the relative roles of stimulusguided mechanisms and endogenous pattern generators in the production of behavior may also vary as conditions change (Fentress, 1972). For instance, a single action might depend on stimulus guidance under certain conditions but shift toward endogenous programming under others. In support of this proposition, Woolridge and Fentress (unpublished observations) found that the ability of a sudden imposed limb displacement to disrupt grooming sequences was reduced during the most rapid phases of grooming. Similarly, Fromm and Evarts (1978) have demonstrated that motor cortex units in rhesus monkeys become relatively insensitive to kinesthetic inputs during rapid ballistic forearm movements in comparison to slower movements of the same limb, and Rushton et al. (1981) have provided similar evidence for humans. As a corollary, reflexcs can also be cnhanced by the higher mechanisms controlling action phase or behavioral state. Illustrations are the potentiation of phasic reflexes in cockroach righting (Camhi, 1977), the potentiation of avoidance reactions to auditory stimuli during flying in crickets (Nolan and Hoy, 1984), or the similar potentiation of trigeminal orienting and jaw opening reflexes in response to predationevoking brain stimulation in cats (Flynn et al., 1971).

These lines of evidence argue clearly that specific reflexes can be both dampened (suppressing the ability of extraneous sensory stimuli to interrupt an action) and enhanced (permitting usually ineffective stimuli to now elicit an action) by higher mecha- 
nisms. However, because studies of simple action generally have adopted behavioral measures that treat actions as all-or-none entities, recording simply their presence or absence, such results can tell us only whether central state changes alter the ability of stimuli to start or stop action. They do not tell us whether sensory stimuli are used differently to guide the form of normal behavior in different action phases or motivational states, whether the rules of integration vary within a given action depending on the context in which it occurs or "whether the same circuits that are reflexly triggered from the periphery might be centrally triggered in patterned sequences" (Bullock, 1977, p. 273 ), producing morphologically similar but mechanistically distinct actions under different conditions. In other words, we do not know whether contextually dependent changes in neural responsiveness simply modify the arousability of action as static entities or whether these changes actually alter the rules by which afferent and central information is integratcd to producc the action.

One means of addressing this question is to improve on allor-none behavioral measures by developing measures that are sensitive to variations in the form of action (Golani, 1976; Golani and Fentress, in press), and to employ these measures with manipulations of sensory processing (such as deafferentation) in different behavioral contexts. If contextually induced changes in sensory processing simply alter the excitability of actions as unit entities, then any changes produced in the form of an action by deafferentation should be constant even when the action appears in different contexts (although the frequency of the action as a whole might vary across contexts). On the other hand, if contextual changes in sensory processing actually alter the integrative circuitry of action production, so that a given action requires specific afferent guidance in some contexts but not in others, then there should be changes in the form of the action after deafferentation that are context-dependent: changes that appear only in certain action phases or under certain motivational conditions but not in others.

The present study employed a fine-grained video analysis of the form of facial and forelimb actions before and after trigeminal deafferentation to investigate the use of trigeminal tactile feedback in action production during ingestive and grooming contexts. This approach was chosen for a number of reasons. First, the trigeminal system provides the afferent limb for a number of well-defined orientation reflexes-jaw opening, etc. (e.g., Flynn et al., 1971; Zeigler et al., 1984). Second, the distribution of trigeminal afferent and efferent fibers into anatomically separate nerves allows the selective removal of oral and facial somatosensory function while preserving all motor function and other sensory function (e.g., gustatory) intact (Jacquin and Zeigler, 1983; Zeigler, 1983). Third, a number of simple actions in which trigeminal tactile feedback might be relevant are seen in both grooming and in taste-elicited behavioral sequences (e.g., tongue protrusions, forelimb facial strokes, forelimb flails, paw licks) (Grill and Berridge, 1985). These actions are identical across the different contexts when examined in a frame-by-frame video analysis. For example, rhythmic tongue protrusions appear with the same protrusion duration and cycle length in both contexts; forelimb flails describe similar trajectories and oscillate at a frequency exceeding $60 \mathrm{~Hz}$ in both contexts, etc. Fourth, the normal temporal proximity of ingestion and grooming sequences in rats (Antin et al., 1975; Bolles, $1960)$ allows the effects of deafferentation on these actions to be compared directly across these different contexts within the same individuals.

\section{Materials and Methods}

\section{Subjects and surgery}

Adult male Sprague-Dawley rats (275-325 gm) were housed in groups of two or three throughout the experiment. To allow ingestive/aversive actions to be elicited and observed, the rats were anesthetized with ketamine $(10 \mathrm{mg} / \mathrm{kg})$ and accpromazine $(1 \mathrm{mg} / \mathrm{kg})$ and implanted with bilateral oral cannulae. These cannulae (heat-flared PE 100 tubing) enter the mouth lateral to the first maxillary molar, are brought out at the dorsal skull, attached to 19-gauge steel tubing, and anchored with skull screws and dental cement (Grill and Norgren, 1978).

Rats served as their own controls: After recovery from surgery, the animals were tested using the procedures described below (over approximately 2 weeks). Eleven rats were then re-anesthetized and subjected to bilateral trigeminal deafferentation (section of the inferior alveolar, lingual, and auriculotemporal nerves of the mandibular branch, and of the anterior superior alveolar and infraorbital nerves of the maxillary branch), following the procedure of Jacquin and Zeigler (1983). Six rats were deafferented unilaterally (three left, three right). Four additional rats were subjected to sham bilateral surgery in which nerves werc exposed but not sectioned. The deafferentation procedurc removes tactile somatosensation and pain from the upper and lower lips, gums, and incisors, anterior tongue, oral mucous membrane, cheek, chin, vibrissae, and facial pads. Chorda tympani gustatory function and trigeminal motor function are spared (Jacquin and Zeigler, 1983; Miller, 1981; Zeigler, 1983). Mandibular nerves were exposed by blunt dissection using the ventral neck approach of Richter (1956), visually identitied under $15 \times$ magnification by comparison with Green (1963), hooked, and sectioned with microscissors. Maxillary nerves were exposed in the orbital canal by gentle orbital deflection, visually identified by comparison with Gregg (1973), hooked, and sectioned (see Jacquin and Zeigler, 1983, for details).

\section{Maintenance}

Following deafferentation, rats were maintained on commercial baby cereal mixed with water to form a loose mash. If body weight fell below $85 \%$ presurgical value (two rats), $10 \mathrm{ml}$ intubations of milk diet (equal parts sweetened condensed milk and water) were given twice daily until body weight recovered to the $85 \%$ value (which occurred within a week). To prevent incisor overgrowth, deafferented rats were anesthetized briefly with halothane once weekly for clipping and sanding of the incisors.

Testing began $48 \mathrm{hr}$ after deafferentation and was completed within 2 weeks, before recovery of function would be expected to occur (Jacquin and Zeigler, 1983; this was confirmed by repeated testing for recovery of facial responsiveness to blunt and sharp probes).

\section{Behavioral criteria}

The focus of this study was on the detailed form of simple actions in taste-elicited and grooming sequences. Actions that are emitted both to palatable taste infusions and in spontaneous grooming bouts include rhythmic tongue protrusions with a cycle length of $166 \pm 7 \mathrm{msec}$ (mean \pm SEM), classified either as midline or as asymmetrical left or right (deviating more than $25^{\circ}$ from midline), and paw or body licking in which tongue protrusions make contact with a body part. Actions emitted both to aversive taste infusions and in grooming sequences include downward forepaw strokes across the face (duration 150-1000 msec depending on stroke amplitude), either with one paw or synchronously with both, with each trajectory classified (1) by amplitude in terms of initial point of forepaw contact [three levels in this study: above the outer corner of the eye (high), between the eye and the rostrolateral border of the mystacial vibrissae (intermediate), and below the vibrissae or nose (low)] and (2) by symmetry (left/right unilateral vs bilateral strokes). Another action shared by aversive and grooming sequences is forelimb flailing, the rapid shaking of one or both forelimbs with a frequency greater than $60 \mathrm{~Hz}$. None of these shared actions differs, either morphologically or temporally, between the two contexts when examined in a frame-by-frame analysis ( $1 / 30 \mathrm{sec}$ resolution). Actions that occurred only in taste-elicited contexts (e.g., rhythmic mouth movements, gapes, chin rubs, and headshakes) were recorded as part of a separate analysis of gustatory reactivity (Berridge and Fentress, 1985).

\section{Test procedure}

Each rat received 12 test trials ( 6 intact, 6 deafferented) on separate days using six different taste stimulus solutions. These solutions were chosen to ensure that a full range of ingestive, aversive, and grooming actions would be elicited. Taste stimuli were sucrose $(1.0$ and $0.03 \mathrm{M}), \mathrm{HCl}(0.1$ and $0.01 \mathrm{M})$, and quinine $\mathrm{HCl}\left(3 \times 10^{-4}\right.$ and $\left.3 \times 10^{-5} \mathrm{M}\right)$. The order of presentation was counterbalanced across rats.

The oral cannulae of a rat were connected to stimulus delivery tubes (PE 50 with PE 10 nozzles), and the rat was placed inside the clear 

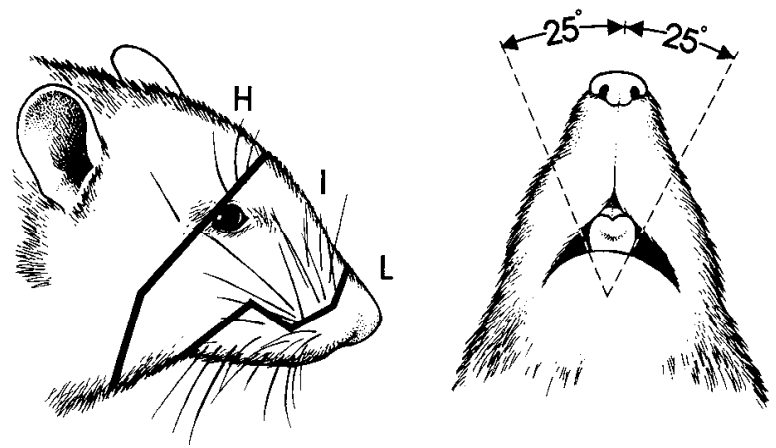

Figure 1. Behavioral scoring criteria. Amplitude zones for forelimb strokes defined by point of initial forelimb contact (left). Tongue protrusion laterality (right). Protrusions in which the outer lingual border remained within the $25^{\circ}$ boundary were considered midline; extensions beyond this zone were classified as asymmetrical.

cylindrical test chamber for $5 \mathrm{~min}$ habituation prior to stimulus infusion. The transparent floor of the test chamber was positioned above a tilted mirror that reflected a view of the rat's face and mouth into a color video camera.

A $1 \mathrm{ml}$ volume of the taste solution was infused into the mouth at a constant rate over $1 \mathrm{~min}$. Taste-elicited responses during the infusion were videotaped for subsequent analysis. The rat was allowed to remain in the test chamber for an additional $6 \mathrm{~min}$ after the infusion. Rats in this situation often groom postprandially; any spontaneous bouts of grooming during this period were similarly videotaped for subsequent analysis.

\section{Behavioral analysis}

A microcomputer was used to assist in the behavioral analysis. Each behavioral action corresponded to a key on the computer keyboard. Additional descriptive modifier keys coded amplitude of response (e.g., extent of tongue protrusion, amplitude of forelimb stroke) and left/right laterality (e.g., asymmetrical vs midline tongue protrusions, unilateral vs bilateral forelimb strokes). Videotapes were scored at $1 / 15$ to $1 / 10$ actual speed by an observer blind to the cxpcrimental condition of the rats. The image of the face filled approximately $50 \%$ of the video screen, allowing sufficient resolution to discriminate varying degrees of amplitude and laterality of tongue protrusions, forelimb strokes, etc. The microcomputer clock was calibrated to the videotape speed. As each action occurred, its corresponding key was pressed together with the appropriate descriptive modifiers. This created a computer transcript of the number, duration, and order of each action. It also created a record of the distribution of each action across variations of amplitude and laterality. This record was stored on floppy disk for subsequent statistical analysis.

\section{Results}

Between-context comparison: influence of ingestive vs grooming contexts

The results are organized by action class comparing intact, unilateral deafferented, bilateral deafferented, and sham-operated groups within ingestive and grooming contexts. Statistical comparisons of action number were made by one-way ANOVA and by the Mann-Whitney $U$ test. Comparisons of proportional distributions were made by ANOVA, and by unpaired $t$ test where $d f=(\operatorname{Var} 1+\operatorname{Var} 2)^{2} /\left((\operatorname{Var} 1)^{2} /(n 1-1)\right)+\left((\operatorname{Var} 2)^{2} /(n 2-1)\right)$ (Ryan et al., 1976).

\section{Forelimb strokes}

Downward forelimb strokes across the face are elicited by aversive taste infusions and emitted spontaneously during postprandial grooming bouts. The absolute number of forelimb strokes in the two contexts did not differ between any of the groups. However, there were marked differences in the distribution of stroke form of bilateral deafferented rats compared to unilaterals or intacts only in the postprandial grooming context $(F(2,28)=16.06, p<0.01 ;$ Fig. 2$)$.

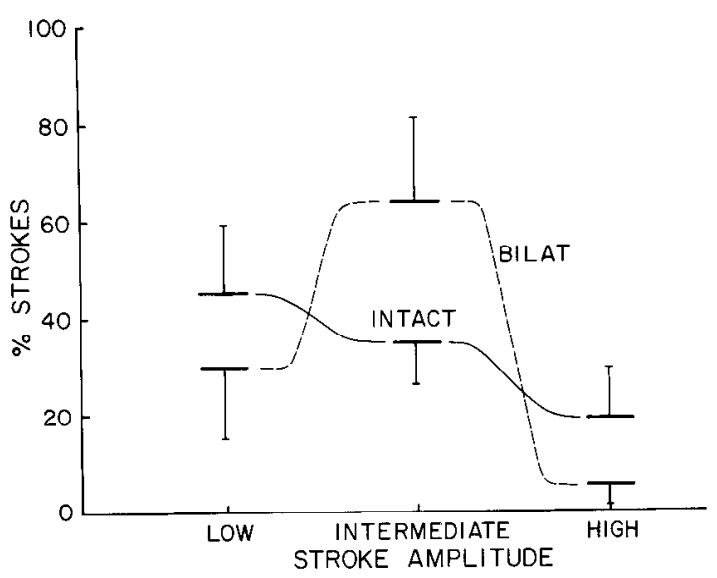

Figure 2. Distribution of forelimb strokes over three levels of amplitude during postprandial grooming. Values refer to the mean percentage $( \pm$ SEM) of total strokes falling within each amplitude category for intact and bilateral deafferented rats.

In aversive face washing, elicited by aversive taste infusions, intact rats distributed strokes with decreasing frequency over low $(56 \%)$, intermediate (34\%), and high (9\%) amplitudes. This distribution did not differ from the aversive strokes of deafferented rats $(F(2,28)=2.38)$. During postprandial grooming, intact (and unilateral) rats increased the proportion of large amplitude strokes but retained the descending ranking of low (45\%), intermediate $(35 \%)$, and high (19\%). The postprandial grooming of bilateral deafferented rats, on the other hand, showed a homogenization of amplitude: Bilaterals emitted a significantly lower proportion of both high amplitude $(t(22)=4.09, p<$ $0.001)$ and low amplitude $(t(15)=2.45, p<0.02)$ strokes and a significantly higher proportion of intermediate strokes $(t(10)=$ $4.6, p<0.001)$ compared to intact rats, resulting in a descending distribution ranking of intermediate $(64 \%)$, low $(30 \%)$, and high $(6 \%)$. Unilateral rats did not differ from intacts in amplitude distribution, but did differ from bilaterals: Bilaterals again showed a lower proportion of low amplitude $(t(11)=2.47, p<0.05)$ and high amplitude $(t(12)=3.14, p<0.01)$ strokes and a greater proportion of intermediate strokes $(t(1)=3.38, p<0.01)$. Unilateral rats did show a greater proportion of postprandial unilateral forelimb strokes (directed to the deafferented side of the face; $t(4)=4.61, p<0.05)$ than did intact $(t(4)=3.58$, $p<0.05)$ or bilateral deafferented $(t(3)=4.15, p<0.05)$ rats. Sham-operated controls did not differ from intacts on any measure.

These data suggest that although the amplitude distribution of normal rats is similar across taste-elicited and postprandial grooming contexts, the mechanisms that produce this distribution are different in the different contexts. Trigeminal feedback is needed from at least one side of the face in order to distribute the amplitude of strokes in postprandial grooming. Aversive face washing, on the other hand, achieves a similar pattern of stroke distribution without need of tactile trigeminal feedback: The pattern of aversive grooming reverts to more central control. Interestingly, the homogeneous grouping of bilateral deafferented strokes about the intermediate point occurs in spite of the fact that our deafferentation procedure spares tactile feedback from the target area of high amplitude strokes.

\section{Forelimb flails}

Forelimb flails are commonly elicited in intact rats by infusions of aversive taste solutions and are occasionally observed in postprandial grooming sequences. During taste infusions, the incidence of forelimb flails did not differ between the groups $(F(2,12)=0.67 ;$ Fig. 3$)$. In postprandial grooming, however, the incidence of flails was highly elevated in bilateral and unilateral 


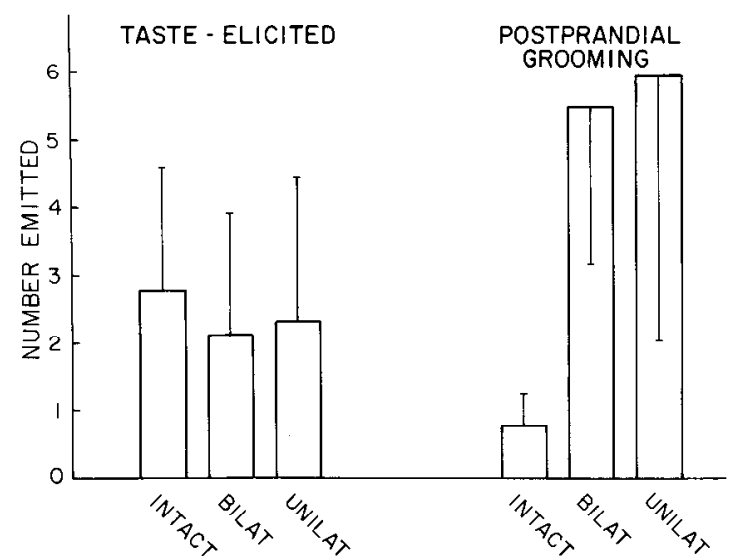

Figure 3. Forelimb flails emitted in ingestive/aversive and postprandial grooming contexts. Values refer to the mean number $( \pm S E M)$ of flails emitted per trial for intact, bilateral deafferented, and unilateral deafferented rats.

deafferented rats compared to intacts $(F(2,14)=5.75, p<0.05)$. Bilateral rats showed significantly more flails during postprandial grooming than did intacts $(p<0.005$; Mann-Whitney $U$ test); unilaterals similarly showed more flails than intacts $(p<$ 0.05 ). Unilaterals did not differ from bilaterals in either context; similarly, sham-operated controls and intacts did not differ.

These data suggest that forelimb flails can be activated either by an aversive taste or by the failure of adequate fecdback following a postprandial stroke. However, the activation during postprandial grooming is not due to the lack of facial tactile input per se nor even simply to the lack of feedback following a stroke (since aversive taste-elicited strokes are not followed by flails), but specifically to the lack of feedback from a stroke while in the grooming context. The lack of feedback following a stroke in the aversive context does not promote flails, providing evidence, in addition to that concerning stroke amplitude, that trigeminal feedback is not used in the control of aversive face washing, but is when strokes are made in the postprandial context.

\section{Tongue protrusions}

Rhythmic tongue protrusions are elicited in intact rats by highly palatable tastes. Rhythmic protrusions at the same frequency are also emitted during facial grooming of intact rats, but the symmetry of the action along the midline is less tightly controlled. Ingestive tongue protrusions of intacts are grouped very closely about the midline (97\%; Fig. 4). During postprandial grooming the proportion of rhythmic protrusions deviating from the midline by more than $25^{\circ}$ increased in intact rats from 3 to $30 \%(t(16)=2.73, p<0.05)$.

The ingestive tongue protrusions of bilateral deafferented rats were significantly more asymmetrical than those of intacts $(t(9)=$ $4.54, p<0.05$ ), suggesting that trigeminal reafference provides negative feedback needed to maintain midline organization during ingestion. Intacts did not differ from bilateral rats during postprandial tongue protrusions, which was largely due to the increased proportion of asymmetrical protrusions shown in this context by intacts. This raises two possibilities: Either trigeminal feedback is not utilized by intact rats to guide postprandial tongue protrusions, or afferent signals are used but in a fashion different from during ingestion (for example, providing positive feedback allowing the lingual tracking of tactile cues). This ambiguity may be resolved by the data from the unilateral deafferented rats, which showed a higher proportion of asymmetrical protrusions than intacts in both ingestive $(t(5)=10.7, p<0.001)$ and postprandial $(t(15)=3.18, p<0.01)$ grooming contexts. The side of asymmetry was not related to the side of deaffer-

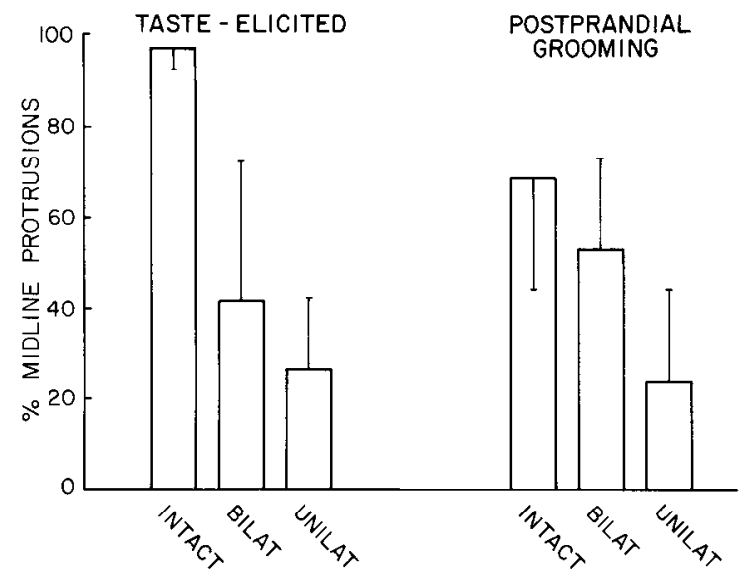

Figure 4. Proportion of midline rhythmic tongue protrusions in ingestive and grooming contexts. Values refer to the mean percentage $( \pm$ SEM $)$ of protrusions within $25^{\circ}$ of midline for intact, bilateral deafferented, and unilateral deafferented rats.

entation. The fact that unilaterals continued to show a higher proportion of asymmetrical protrusions than intacts even during postprandial grooming implies that feedback is not irrelevant during postprandial rhythmic protrusions (or else the groups would not differ) but rather may be used differently in the two contexts.

\section{Within-context comparison: effects of phase within the grooming context}

Within the behavioral category of postprandial facial grooming in mice are discrete phases, defined by the sequential relations between the actions (Fentress and Stillwell, 1973). In rats, we have identified two main phases: sequentially variable, comprising the bulk of grooming, and sequentially stereotyped, in which a fixed rule of order is followed (Berridge and Fentress, 1984). The constituent actions (paw licking, forelimb strokes of varied amplitude, head tucking, and body licking) are very similar in the two phases. However, in the variable phase the actions occur in flexible and largely unpredictable order; in the sequentially stereotyped phase a comparatively rigid pattern of order is followed: a set of six to eight rapid elliptical $1 \mathrm{~cm}$ diameter forepaw strokes $(6-7 \mathrm{~Hz})$ over the mystacial vibrissae, followed by a short series of slower strokes of ascending amplitude, with the final strokes passing generally above and caudal to the pinna, followed by a tucking of the head and twisting of the body, and terminating with licking of the ventrolateral torso (Fig. 5). This entire chain typically is completed by the initiation of body licking within $5 \mathrm{sec}$. It is often seen in the postprandial grooming of intact rats but rarely during taste-elicited face washing.

This stereotyped chain persisted after bilateral trigeminal deafferentation. In addition to the survival of the overall sequential structure of this phase, most of the deformations of individual actions that characterized the postprandial grooming of bilateral deafferented rats as a whole were found explicitly not to exist in actions within this phase. For instance, the homogenization of deafferented stroke amplitude was absent in sequentially stereotyped grooming: During this phase, bilateral deafferented rats showed a distribution of low-, intermediate-, and high-amplitude strokes similar to that of intacts. In fact, the majority of high-amplitude strokes seen in bilateral deafferented postprandial grooming occurred in this phase, in marked contrast to intact rats. Similarly, the frequent disruption of grooming by forelimb flails, which characterized deafferented postprandial grooming as a whole, did not occur during this phase. Flails were rarely observed during the sterotyped phase and, when they did occur, virtually never terminated nor disrupted the sequential order. 
STEREOTYPED SEQUENCE PHASE
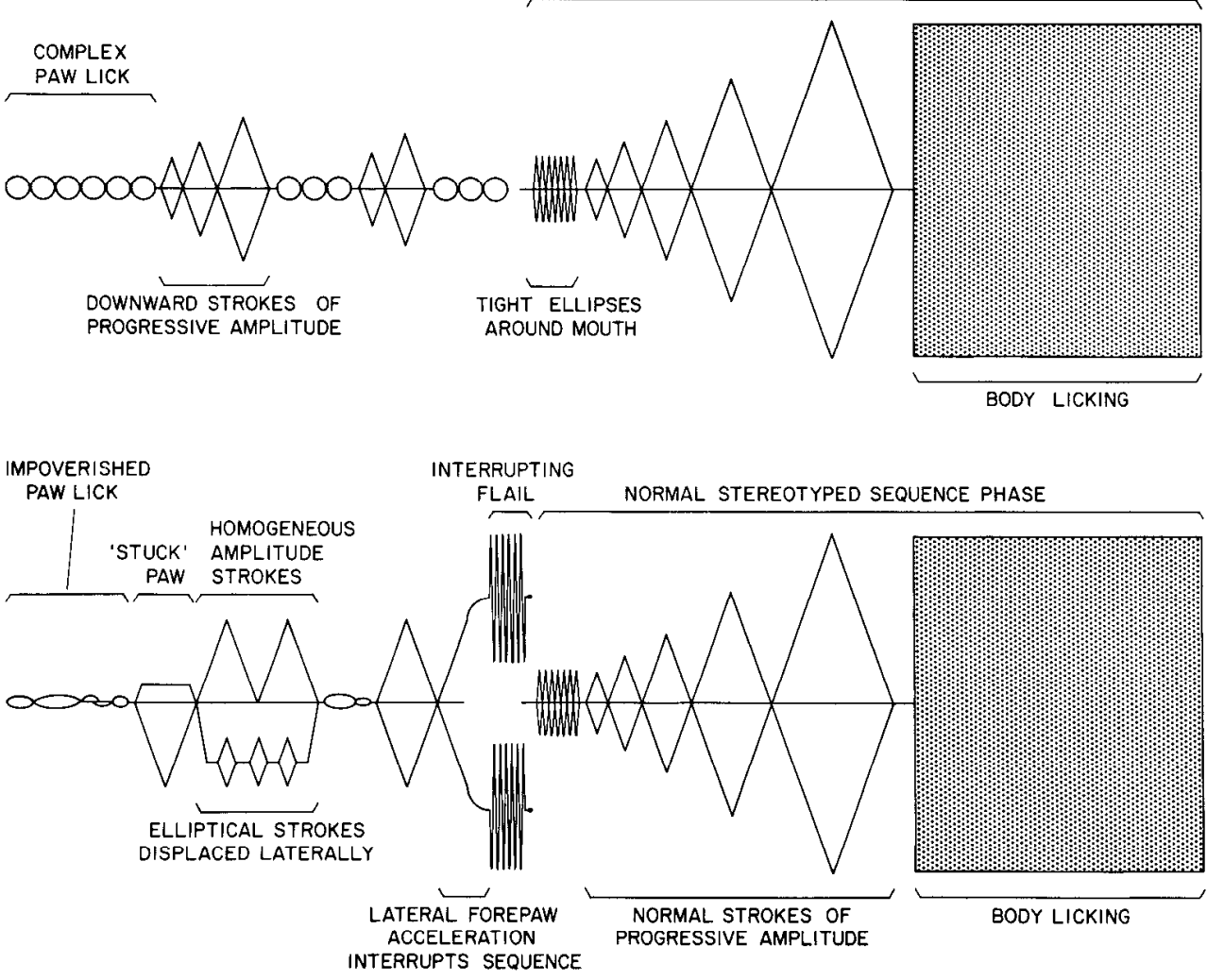

Figure 5. Idealized postprandial grooming sequences of intact (top) and bilateral deafferented (bottom) rats. Deviations of the lines above and below the horizontal axis represent the trajectories of the forepaws from the nose along the face.
These observations indicate that the occurrence of fixed or sequentially stereotyped chains of facial grooming is not the product of a cascading series of reflexes to tactile trigeminal stimuli. Further, it is not only that the order of action is specified endogenously during stereotyped phases, but also that the mechanisms controlling individual action form are switched from stimulus-guided to more centrally guided modes during this phase. Forelimb stroke amplitude can be specified without need of somatosensory feedback during the sequentially stereotyped, but not during the sequentially variable, grooming phase. The integrative circuitry that produces the pattern of this action is functionally modified in the different phases of grooming.

\section{Additional action changes}

In addition to the various context-dependent changes described above, a number of action changes observed after deafferentation appeared in several behavioral contexts. The precise alterations in movement organization were often idiosyncratic to each individual, thus making a systematic catalog of these "clinical" syndromes difficult. However, certain common elements were noted.

During forelimb strokes involving downward trajectories across the face, limbs on the deafferented side(s) of the rat were likely to be brought against the face with excessive pressure. This often caused the forelimb to become either "stuck" momentarily in one spot or to "bounce" laterally away from the face. When this happened the subsequent trajectories of the two paws could show a momentary but marked spatiotemporal asymmetry.

Paw licking in deafferented rats was characterized by poor coordination between paw and tongue movements, with the result that the tongue often missed the paws entirely. In unilateral deafferented rats, the paw and digits of the forelimb on the intact side of the face were both more active and more clearly oriented toward the tongue compared to the paw on the deafferented side. Indeed, we were often struck by the illusion, in several rats, that the forelimb itself had been deafferented.

\section{Discussion}

The results of the present study show that the use of sensory feedback in the control of simple action form varies as a function of the behavioral context in which the action occurs. An action that is formed in one context through an integration of afferent signals with central specifications, and that is deformed in that context by deafferentation, is produced in another context by a functionally different mechanism, which requires no tactile feedback and is unaffected by deafferentation. For instance, the control of forelimb stroke amplitude requires tactile feedback from the face during postprandial grooming but not when the same strokes are activated during taste-elicited aversion. Even in postprandial grooming itself, stroke amplitude can be specified without somatosensory feedback and is during sequentially stereotyped grooming phases but is not during sequentially variable phases. Second, rhythmic tongue protrusions during ingestion require tactile feedback to maintain midline orientation and are asymmetrically distorted by deafferentation. Rhythmic tongue protrusions emitted during postprandial grooming are no longer maintained on the midline by negative feedback. Third, the failure of a forelimb stroke to produce normal feedback during the sequentially variable phase of postprandial grooming results in an interrupting forelimb flail and a pause in grooming, but does not when the stroke is emitted during either aversive face washing or the sequentially stereotyped phase of postprandial grooming. The contextual sensitivity of these deafferentationinduced behavioral changes is striking. Contextual influences are compatible with existing electrophysiological data on the variable sensitivity of tactile, proprioceptive, visual, and gustatory neural systems (Chang and Scott, 1984; Manetto and Lidsky, 1984; Rolls et al, 1979), if one assumes that such electrophysiological changes may reflect a functional reorganization of the neural circuitry involved in producing and coordinating action (Evarts et al., 1984).

The existence of contextually dependent changes in the structure and integrative neural circuitry of simple action carries 
implications for our concepts of neural control hierarchies. In its purest form, a hierarchical mechanism creates complex behavior by recruiting simpler action mechanisms as units, to form the basic building blocks of coordinated patterns (Bullock, 1977; Easton, 1972; Gallistel, 1980). By a strictly hierarchical model, the higher-order mechanisms corresponding to the initiation of ingestion or grooming call up particular actions, such as tongue protrusions or forelimb strokes, by potentiating them as entire entities without specifiying the actual detail of those actions. If a particular simple action is activated in more than one context, its intrinsic organization should not depend on which higher mechanism potentiated it (Gallistel, 1980). Our discovery of context-specific changes in the form of natural actions after trigeminal deafferentation suggests that these actions, which are controlled essentially by spinal and brain stem mechanisms (Grill and Norgren, 1978), do not correspond to simple production units. This could indicate either a nonhierarchical control of production (Fentress, 1984) or that even these relatively simple actions are actually composites of multiple production units whose control hierarchies only partially overlap in different contexts (Dawkins, 1976; Gallistel, 1980).

In either case, the existence of context-specific changes in the form of natural actions after deafferentation illustrates the importance of higher-order variables such as context and phase in the control of vertebrate action (cf. Evarts et al., 1984). Motivational "sets," which potentiate entire classes of action such as ingestion or natural grooming, or even subsets within a single context (e.g., the flexible versus the chain phases of grooming), are by no means mere epiphenomena, but are instead real control factors that exert a powerful descending influence over sensorimotor integration.

\section{References}

Antin, J., J. Gibbs, J. Holt, R. C. Young, and G. P. Smith (1975) Cholecystokinin elicits the complete behavioral sequence of satiety in rats. J. Comp. Physiol. Psychol. 89: 784-790.

Berridge, K. C., and J. C. Fentress (1984) Trigeminal deafferentation and control of ingestive and grooming sequences in rats. Neurosci. Abstr. 10: 398 .

Berridge, K C., and J. C. Fentress (1985) Trigeminal-taste interaction in palatability processing. Science $228: 747-750$.

Bolles, R. C. (1960) Grooming behavior in the rat. J. Comp. Physiol. Psychol. 53: 306-310.

Bullock, T. H., with R. Orkand and A. Grinnell (1977) Introduction to Nervous Systems, Freeman, San Francisco.

Camhi, J. M. (1977) Behavioral switching in cockroaches: Transformation of tactile reflexes during righting behavior. J. Comp. Physiol. 113:283-301

Chang, F.-C. T., and P. R. Scott (1984) Conditioned taste aversions modify neural responses in the rat nucleus tractus solitarious. $J$. Ncurosci. 4: 1850-1862.

Chapin, J. K., and D. J. Woodward (1981) Modulation of sensory responsiveness of single somatosensory cortical cells during movement and arousal behavior. Exp. Neurol. 72: 164-178.

Dawkins, R. (1976) Hierarchical organization: A candidate principle for ethology. In Growing Points in Ethology, P. P. G. Bateson and R. A. Hinde, eds., pp. 7-54, Cambridge, Cambridge, UK.

Doty, R. W., Sr. (1976) The concept of neural centers. In Simpler Networks and Behavior, J. C. Fentress, ed., pp. 251-265, Sinauer, Sunderland, MA.

Easton, T. A. (1972) On the normal use of reflexes. Am. Sci. 60:591599.

Evarts, E. V., Y. Shinoda, and S. P. Wise (1984) Neurophysiological Approaches to Higher Brain Functions, Wiley, New York.

Ewert, J.-P. (1985) Concepts in vertebrate neuroethology. Anim. Behav. 33: 1-29.

Fentress, J. C. (1972) Development and patterning of movement sequences in inbred mice. In The Biology of Behavior, J. Kiger, ed., pp. 83-132, Oregon State University, Corvallis, OR.
Fentress, J. C. (1984) The development of coordination. J. Motor Behav. 16: $99-134$

Fentress, J. C., and F. P. Stilwell (1973) Grammar of a movement sequence in inbred mice. Nature 244: 52-53.

Flynn, J. P., S. B. Edwards, and R. J. Bandler (1971) Changes in sensory and motor systems during centrally elicited attack. Behav. Sci. 16: 119.

Fromm, C., and E. V. Evarts (1978) Motor cortex responses to kinesthetic inputs during postural stability, precise fine movement and ballistic movement in the conscious monkey. In Active Touch, G. Gordon, ed., pp. 105-117, Pergamon, Oxford, UK.

Gallistel, C. R. (1980) The Organization of Action, Erlbaum, Hillsdale, NJ.

Gardner, E. (1968) Fundamentals of Neurology (5th Ed.), Saunders, Philadelphia.

Golani, I. (1976) Homeostatic motor processes in mammalian interactions: A choreography of display. In Perspective in Ethology: Vol. 2, P. P. G. Bateson and P. H. Klopfer, eds., Plenum, New York.

Golani, I., and J. C. Fentress (in press) Ontogeny of face grooming in mice. Dev. Psychobiol.

Gregg, J. M. (1973) A surgical approach to the ophthalmic-maxillary nerve trunks in the rat. J. Dent. Res. 52: 392

Green, E. C. (1963) Anatomy of the Rat, Hafner, New York.

Grill, H. J., and K. C. Berridge (1985) Taste reactivity as a measure of the neural control of palatability. In Progress in Psychobiology and Physiological Psychology, 1985, Vol. 11, J. M. Sprague and A. N. Epstein, eds., Academic, New York.

Grill, H. J., and R. Norgren (1978) The taste reactivity test: I and II Brain Res. 143: 263-297.

Hoyle, G. (1964) Exploration of neuronal mechanisms underlying behavior in insects. In Neural Theory and Modeling, R. F. Reiss, eds., pp. 346-376, Stanford, Stanford, CA.

Jacquin, M. F., and H. P. Zeigler (1983) Trigeminal orosensation and ingestive behavior in the rat. Behav. Neurosci. 97: 62-97.

Kimmel, C. B., and R. C. Eaton (1976) Development of the Mauthner cell. In Simpler Networks and Behavior, J. C. Fentress, ed., pp. 186202, Sinauer, Sunderland, MA.

Kugelberg, E. (1952) Facial reflexes. Brain 75: 385-396.

Manetto, C., and T. I. Lidsky (1984) The effects of movements on the sensory responsiveness of basal ganglia units. Neurosci. Abstr. 10: 350.

Miller, M. G. (1981) Trigeminal deafferentation and ingestive behavior in the rat. J. Comp. Physiol. Psychol. 95: 252-269.

Nolan, T. G., and R. R. Hoy (1984) Initiation of behavior by single neurons: The role of behavioral context. Science 226: 992-994.

Pinsker, H., I. Kupfermann, V. Castellucci, and E. R. Kandel (1970) Habituation and dishabituation of the gill-withdrawal reflex in Aplysia. Science 167: 1740-1742.

Richter, C. P. (1956) Salt appetite of mammals: Its dependence on instinct and metabolism. L'Instinct dans le Comportement des Animaux et de l'Homme, M. Autori, ed., Masson, Paris.

Rolls, E. T., S. J. Thorpe, S. Maddison, A. Roper-Hall, A. Puerto, and D. Perrett (1979) Activity of neurones in the neostriatum and related structures in alert animals. In The Neostriatum, I. Divac and R. Oberg, eds., Pergamon, Oxford.

Rushton, D. N., J. C. Rothwell, and M. D. Craggo (1981) Gaiting of somatosensory evoked potentials during different kinds of movement in man. Brain 104: 465-491.

Ryan, T. A., B. L. Joiner, and B. F. Ryan (1976) Minitab Student Handbook, Duxbury, North Scituate, MA.

Sherrington, C. S. (1906) The Integrative Action of the Nervous System, Yale, New Haven, CT.

Stent, G. S., W. B. Kristan, W. D. Friesen, C. A. Ort, M. Poon, and R. C. Calabrese (1978) Neuronal generation of the leech swimming movement. Science 200: 1348-1357.

Wilson, D. M. (1961) The central nervous control of flight in a locust. J. Exp. Biol. 38: 471-490.

Zeigler, H. P. (1983) The trigeminal system and ingestive behavior. In Handbook of Bchavioral Neurobiology: Vol. 6-Motivation, E. Satinoff and P. Teitlebaum, eds., Plenum, New York.

Zeigler, H. P., K. Semba, and M. F. Jacquin (1984) Trigeminal reflexes and ingestive behavior in the rat. Behav. Neurosci. 98: 1023-1038. 